\title{
EMBEDDING A NON-EMBEDDABLE STABLE PLANE
}

ABSTRACT. In [4], K. Strambach describes a 2-dimensional stable plane $\mathscr{P}_{\mathbb{R}}$ admitting $\Sigma=\mathrm{SL}_{2} \mathbb{R}$ as a group of automorphisms such that there exists no $\Sigma$-equivarient embedding into a 2 dimensional projective plane. $R$. Löwen [3] has given a 4-dimensional analogue $\mathscr{S}_{\mathbb{C}}$, admitting $\Delta=\mathrm{SL}_{2} \mathrm{C}$. He posed the question whether there are embeddings of Strambach's plane $\mathscr{S}_{\mathbb{R}}$ into $\mathscr{Y}_{\mathbb{C}}$. We show that such embeddings exist, in fact we determine all $\Sigma$-equivariant embeddings of 2-dimensional stable planes admitting $\Sigma$ as a transitive group of automorphisms.

\section{The PLANES}

In the original definitions, the point space is taken to be $\mathbb{R}^{2}$ or $\mathbb{C}^{2}$, respectively, and the lines are described as subsets of the point space. We wish to describe the resulting geometries by the method given in [6]. Since this method applies only to point homogeneous geometries, we have to delete the origin.

(1.1) NOTATION. We write

$$
\begin{aligned}
& \Sigma=\mathrm{SL}_{2} \mathbb{R}=\left\{\left(\begin{array}{ll}
a & b \\
c & d
\end{array}\right) \mid a, b, c, d \in \mathbb{R} ; a d-b c=1\right\} \\
& \Delta=\mathrm{SL}_{2} \mathbb{C}=\left\{\left(\begin{array}{ll}
a & b \\
c & d
\end{array}\right) \mid a, b, c, d \in \mathbb{C} ; a d-b c=1\right\} .
\end{aligned}
$$

The following stabilizers (with respect to the linear actions on $\mathbb{R}^{2}$ and $\mathbb{C}^{2}$ ) shall be useful:

$$
\begin{aligned}
& \Pi=\Sigma_{(1,1)}=\left\{\left(\begin{array}{cc}
1-t & -t \\
t & 1+t
\end{array}\right) \mid t \in \mathbb{R}\right\}=\left\{\left(\begin{array}{ll}
1 & \\
t & 1
\end{array}\right) \mid t \in \mathbb{R}\right\}^{\left(\begin{array}{l}
11 \\
1
\end{array}\right)} \\
& \left.\Phi=\Delta_{(1, i)}=\left\{\left(\begin{array}{cc}
1-i z & z \\
z & 1+i z
\end{array}\right) \mid z \in \mathbb{C}\right\}=\left\{\left(\begin{array}{ll}
1 & \\
z & 1
\end{array}\right) \mid z \in \mathbb{C}\right\}^{\left({ }^{1} i\right.}{ }_{i}\right)
\end{aligned}
$$

For the description of the planes that are of interest in this paper, we consider the following sets of points:

$$
\begin{aligned}
& R_{o}=\{(x, x) \mid x \in \mathbb{R}\}, R_{e}=\{(1, y) \mid y \in \mathbb{R}\}, R_{s}=\{(x, 1 / x) \mid x>0\} \\
& S_{o}=\{(u, i u) \mid u \in \mathbb{C}\}, S_{e}=\{(1, v) \mid v \in \mathbb{C}\}, \\
& S_{s}=\{(x, i / x+y) \mid x, y \in \mathbb{R}, x>0\}
\end{aligned}
$$


and the corresponding stabilizers:

$$
\begin{aligned}
\Lambda_{o} & =\Sigma_{R_{o}}=\left\{\left(\begin{array}{cc}
s-t & s-1 / s-t \\
t & 1 / s+t
\end{array}\right) \mid s, t \in \mathbb{R} ; s \neq 0\right\} \\
& \left.=\left\{\left(\begin{array}{ll}
s & \\
t & 1 / s
\end{array}\right) \mid s, t \in \mathbb{R} ; s \neq 0\right\}^{(1)}\right) \\
\Lambda_{e} & =\Sigma_{R_{e}}=\left\{\left(\begin{array}{cc}
1 & t \\
& 1
\end{array}\right) \mid t \in \mathbb{R}\right\}, \Lambda_{s}=\Sigma_{R_{s}}=\left\{\left(\begin{array}{cc}
a & 1 / a
\end{array}\right) \mid a>0\right\} \\
\Omega_{o} & =\Delta_{S_{o}}=\left\{\left(\begin{array}{cc}
u-i z & i(u-1 / u-i z) \\
z & 1 / u+i z
\end{array}\right) \mid u, z \in \mathbb{C} ; u \neq 0\right\} \\
\Omega_{e} & =\Delta_{S_{e}}=\left\{\left(\begin{array}{cc}
1 & z \\
& 1
\end{array}\right) \mid z \in \mathbb{C}\right\}, \\
\Omega_{s} & =\Delta_{S_{s}}=\left\{\left(\begin{array}{cc}
a & b \\
& 1 / a
\end{array}\right) \mid a, b \in \mathbb{R} ; a>0\right\} .
\end{aligned}
$$

Note that, for each $k \in\{o, e, s\}$, we have that $R_{k}=(1,1) \Lambda_{k}$, and $S_{k}=(1, i) \Omega_{k}$. (1.2) PROPOSITION. For cosets $\mathrm{A} \alpha, \mathrm{B} \beta$ (where $\mathrm{A} \in\{\Pi, \Phi\}, \mathrm{B} \in\left\{\Lambda_{o}, \Lambda_{e}\right.$, $\left.\Lambda_{s}, \Omega_{o}, \Omega_{e}, \Omega_{s}\right\}$ ) define the relation

$$
\mathrm{A} \alpha \mathrm{I} \mathrm{B} \beta \Leftrightarrow \mathrm{A} \alpha \cap \mathrm{B} \beta \neq \varnothing .
$$

Then the incidence geometries

$$
\begin{array}{ll}
\left(\Sigma / \Pi, \Sigma / \Lambda_{o} \cup \Sigma / \Lambda_{e}, I\right) & \left(\Sigma / \Pi, \Sigma / \Lambda_{o} \cup \Sigma / \Lambda_{s}, I\right) \\
\left(\Delta / \Phi, \Delta / \Omega_{o} \cup \Delta / \Omega_{e}, I\right) & \left(\Delta / \Phi, \Delta / \Omega_{o} \cup \Delta / \Omega_{s}, I\right)
\end{array}
$$

are isomorphic with the real affine plane $\mathscr{A}_{\mathbb{R}}$, Strambach's plane $\mathscr{S}_{\mathbb{R}}$, the complex affine plane $\mathscr{A}_{\mathrm{C}}$, and Löwen's plane $\mathscr{S}_{\mathrm{C}}$, respectively (each minus the origin).

Proof. For $\mathbb{K} \in\{\mathbb{R}, \mathbb{C}\}$, the group $\mathrm{SL}_{2} \mathbb{K}$ acts transitively on the points of $\mathscr{A}_{K}$ and $\mathscr{I}_{\mathbb{K}}$ (minus the origin). It is easy to see that in each of the considered cases there are exactly two line orbits: namely, the line pencil in the origin and the complement thereof (for $\mathscr{S}_{\mathbb{K}}$ this holds by definition), and that the stabilizer of any point $p$ acts with two orbits on the line pencil in $p$. Thus the orbit decomposition of the pencil (under the action of the stabilizer) coincides with the decomposition that is induced from the action of $\mathrm{SL}_{2} \mathbb{K}$ on the line space. Obviously, the stabilizers of representatives for the different orbits are different. Thus [6:4] yields the assertion. 
(1.3) PROPOSITION. Assume that $\mathscr{S}$ is a linear space (resp., a stable plane) admitting a group $\Gamma$ of automorphisms such that the reconstruction method of [6] applies; i.e. the group $\Gamma$ acts transitively on the point set, and there is a cross-section $\mathscr{R}$ for the line orbits that simultaneously forms a cross-section for the orbits in the line pencil through some point $p$ under the stabilizer $\Gamma_{p}$.

If $\Gamma$ acts point-transitively on a linear space (resp., a stable plane) $\mathscr{S}^{\prime}$ such that

- for each $R \in \mathscr{R}$ there is a line $R^{\prime}$ of $\mathscr{S}^{\prime}$ such that $\Gamma_{R}=\Gamma_{R^{\prime}}$,

-there is a point $p^{\prime}$ of $\mathscr{S}^{\prime}$ that is incident with each of the $R^{\prime}$, and $\Gamma_{p}=\Gamma_{p^{\prime}}$ then $\mathscr{S}$ and $\mathscr{S}^{\prime}$ are isomorphic, and the actions of $\Gamma$ are equivalent.

Proof. According to [6], the linear space $\mathscr{S}$ is isomorphic with the incidence geometry whose point space is the orbit $p^{\prime \Gamma}$ and whose line space is $\bigcup_{R \in \mathscr{R}} R^{\prime}$. Therefore we just have to show that the line space of $\mathscr{S}^{\prime}$ equals the union of the orbits $R^{\prime \Gamma}$, where $R \in \mathscr{R}$. Because joining lines are unique in $\mathscr{S}^{\prime}$, this follows immediately from the fact that two points of $\mathscr{S}$ are joined by a line in $\bigcup_{R \in \mathscr{R}} R^{\Gamma}$.

To recognize Strambach's plane, we shall need the following:

(1.4) LEMMA. Let $\Sigma, \Pi$ and $\Lambda_{o}$ be as in (1.1), and write

$$
\Lambda(t)=\left\{\left(\begin{array}{cc}
a & t(a-1 / a) \\
1 / a
\end{array}\right) \mid a>0\right\} \text { for each } t \in \mathbb{R} .
$$

With the incidence relation I as in (1.2), the incidence structure $\left(\Sigma / \Pi, \Sigma / \Lambda_{o} \cup \Sigma / \Lambda(t), \mathrm{I}\right)$ is isomorphic with $\mathscr{S}_{\mathbb{R}}$ for each choice of $t \in \mathbb{R} \backslash\{1\}$.

Proof. Conjugation with $\left(\begin{array}{cc}1 & t \\ & 1-t\end{array}\right) \in \mathrm{GL}_{2} \mathbb{R}$ leaves both $\Pi$ and $\Lambda_{o}$ invariant, but carries $\Lambda_{s}$ to $\Lambda(t)$. In view of (1.2) and [6], this yields the assertion.

(1.5) REMARKS. (a) The 2-dimensional stable planes admitting $\mathrm{SL}_{2} \mathbb{R}$ as a group of automorphisms have been determined by R. Löwen [2]. Among these, the punctured real affine and the punctured Strambach plane are the only ones where $\mathrm{SL}_{2} \mathbb{R}$ acts point-transitively.

(b) There is no analogue to $\mathscr{S}_{\mathbb{R}}$ and $\mathscr{S}_{\mathbb{C}}$ over Hamilton's quaternions [5].

We shall not use this classification of $\mathrm{SL}_{2} \mathbb{R}$-planes, since our special situation allows to identify the planes that are isomorphic with the punctured real affine plane or the punctured Strambach plane by means of (1.3) and (1.4). 


\section{ORBITS}

As a subgroup of $\Delta$, the group $\Sigma$ has a natural action on $\mathbb{C}^{2}$. We characterize the orbits:

(2.1) LEMMA. For $(u, v) \in \mathbb{C}^{2} \backslash\{(0,0)\}$, one of the following holds:

(a) There is some $w \in \mathbb{C}^{*}$ such that $w(u, v) \in \mathbb{R}^{2}$. Then the orbit $(u, v) \Sigma$ has dimension 2, and the action of $\Sigma$ is equivalent to the natural action on $\mathbb{R}^{2} \backslash\{(0,0)\}=(1,1) \Sigma$. Moreover, the orbit $(u, v) \Sigma$ is closed in $\mathbb{C}^{2} \backslash\{(0,0)\}$, hence locally compact.

(b) For each $w \in \mathbb{C}^{*}$, the vector $w(u, v)$ does not belong to $\mathbb{R}^{2}$. Then the orbit $(u, v) \Sigma$ has dimension 3 , and $\Sigma$ acts freely on that orbit.

Proof. We have to determine the stabilizer $\Sigma_{(u, v)}$. Since $\Sigma$ acts $\mathbb{C}$-linearly, we have that $\Sigma_{(u, v)}=\Sigma_{w(u, v)}$ for each $w \in \mathbb{C}^{*}$, and scalar multiplication with $w$ is a homeomorphism from $\mathbb{C}^{2} \backslash\{(0,0)\}$ onto itself that carries $(u, v) \Sigma$ to $w(u, v) \Sigma$. This implies assertion (a) (recall that $\Sigma$ acts transitively on $\left.\mathbb{R}^{2} \backslash\{(0,0)\}\right)$. In the situation of (b), we may assume that $(u, v)=(1, v)$ for some $v \in \mathbb{C} \backslash \mathbb{R}$. Then 1 and $v$ form an $\mathbb{R}$-basis for $\mathbb{C}$. The stabilizer condition $(1, v)=(a+v c, b+v d)$ yields that $\left(\begin{array}{ll}a & b \\ c & d\end{array}\right)=\left(\begin{array}{ll}1 & \\ & 1\end{array}\right)$, since $a, b, c, d \in \mathbb{R}$. This proves assertion (b).

\section{EQUIVARIANT EMBEDDINGS}

(3.1) DEFINITION. Let $\mathscr{S}$ be a 2-dimensional stable plane, and assume that $\Sigma=\mathrm{SL}_{2} \mathbb{R}$ acts effectively and transitively on $\mathscr{S}$. A $\Sigma$-equivariant embedding of $\mathscr{S}$ into $\mathscr{S}_{\mathbb{C}}$ is a triplet $(\pi, \lambda, \gamma)$ with the property that

$-\pi$ and $\lambda$ are embeddings of the point and line space of $\mathscr{S}$ into the point and line space of $\mathscr{S}_{\mathbb{C}}$, respectively;

$-(\pi, \lambda)$ is a collineation onto $\mathscr{S}^{(\pi, \lambda)}$;

$-\gamma$ is a continuous monomorphism of $\Sigma$ into $\Delta$ such that the diagram

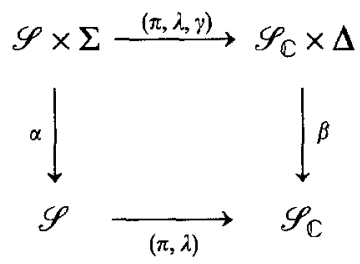

commutes, where $\alpha$ and $\beta$ denote the action of $\Sigma$ and $\Delta$, respectively. 
(3.2) In the sequel, we shall consider the case where $\gamma=0$. According to (2.1) we may assume that the image of $\pi$ equals $w(s, t) \Sigma$ for some $w \in \mathbb{C}^{*}$ and $(s, t) \in \mathbb{R}^{2} \backslash\{(0,0)\}$. Adapting $(s, t)$, one obtains that $w \bar{w}=1$. Now there is $\sigma \in \Sigma$ such that $(s, t) \sigma=(1,1)$, and we may assume that the image of $\pi$ equals $(w, w) \Sigma$ for some $w=\mathbb{C}^{*}$ with $w \bar{w}=1$.

(3.3) We write

$$
\varphi=\left(\begin{array}{cc}
w & w-i \bar{w} \\
\bar{w}
\end{array}\right) \in \Delta, \quad \text { hence }(1, i) \varphi=(w, w) .
$$

To determine the incidence structure on $\mathscr{S}^{(\pi, \lambda)}$ with the help of (1.3) we put $p=(1,1), \quad \mathscr{R}=\left\{L_{o}, L_{e}\right\} \quad$ resp. $\mathscr{R}=\left\{L_{o}, L_{s}\right\} \quad$ and $\quad p^{\prime}=(1, i) \varphi=(w, w)$, $L_{o}^{\prime}=S_{o} \varphi, L_{k}^{\prime}=S_{s} \varphi$ for $k=e$ or $k=s$, respectively. Now we are able to compute the stabilizers $\Sigma_{x}$ (for $x \in\left\{p^{\prime}, S_{o} \varphi, S_{s} \varphi\right\}$ ) in the following way:

$$
\Sigma_{x}=\Sigma \cap \Delta_{x}=\Sigma \cap \Delta_{x \phi}^{\phi}{ }^{-1} .
$$

Observe that

$$
\left(\begin{array}{cc}
\bar{w} & i \bar{w}-w \\
& w
\end{array}\right)\left(\begin{array}{cc}
w & w-i \bar{w} \\
& \bar{w}
\end{array}\right)=\left(\begin{array}{ll}
1 & \\
& 1
\end{array}\right) .
$$

(3.4) For the action (via conjugation) of $\varphi$ on $\Delta$ we obtain

$\left(\begin{array}{ll}a & b \\ c & d\end{array}\right)^{\varphi}=\left(\begin{array}{cc}a-w(w-i \bar{w}) c & \bar{w}(w-i \bar{w}) a-(w-i \bar{w})^{2} c+\bar{w}^{2} b-\bar{w}(w-i \bar{w}) d \\ w^{2} c & w(w-i \bar{w}) c+d\end{array}\right)$.

(3.5) Applying (3.4) to elements of $\Phi$ we get

$$
\left(\begin{array}{cc}
1-i z & z \\
z & 1+i z
\end{array}\right)^{\varphi}=\left(\begin{array}{cc}
1-w^{2} z & -w^{2} z \\
w^{2} z & 1+w^{2} z
\end{array}\right)
$$

Therefore,

$$
\Sigma_{(w, w)}=\Phi^{\varphi} \cap \Sigma=\left\{\left(\begin{array}{cc}
1-t & -t \\
t & 1+t
\end{array}\right) \mid t \in \mathbb{R}\right\}=\Pi .
$$

(3.6) For the elements of $\Omega_{o}$ we obtain:

$$
\left(\begin{array}{cc}
u-i z & i(u-1 / u-i z) \\
z & 1 / u+i z
\end{array}\right)^{\varphi}=\left(\begin{array}{cc}
u-w^{2} z & u-1 / u-w^{2} z \\
w^{2} z & 1 / u+w^{2} z
\end{array}\right)
$$

Therefore $\Sigma_{S_{o} \varphi}=\Omega_{o}^{\varphi} \cap \Sigma=\Lambda_{o}$.

(3.7) Finally, we turn the elements of $\Omega_{s}$ :

$$
\left(\begin{array}{cc}
a & b \\
& 1 / a
\end{array}\right)^{\varphi}=\left(\begin{array}{cc}
a & \bar{w}^{2} b+\left(1-i \bar{w}^{2}\right)(a-1 / a) \\
1 / a
\end{array}\right) .
$$


If $\bar{w}^{2} \in \mathbb{R} \quad$ (i.e. if $\left.w \in\{1,-1, i,-i\}\right)$ then the imaginary part of $\bar{w}^{2} b+\left(1-i \bar{w}^{2}\right)(a-1 / a)$ equals $-i \bar{w}^{2}(a-1 / a)$ (recall that $\left.a, b \in \mathbb{R}\right)$. If $\left(\begin{array}{cc}a & b \\ & 1 / a\end{array}\right) \in \Sigma$, we infer that $a=1$. Hence $\Sigma_{S_{s} \varphi}=\Omega_{s}^{\varphi} \cap \Sigma=\Lambda_{e}$.

If $\bar{w}^{2} \in \mathbb{C} \backslash \mathbb{R}$, we write $\bar{w}^{2}=x+i y$, where $x, y \in \mathbb{R}$ and $y \neq 0$. Then

$$
\bar{w}^{2} b+\left(1-i \bar{w}^{2}\right)\left(a-\frac{1}{a}\right) \in \mathbb{R}
$$

if and only if

$$
\bar{w}^{2} b-i \bar{w}^{2}\left(a-\frac{1}{a}\right) \in \mathbb{R} .
$$

This implies that $y b-x(a-1 / a)=0$. Hence $b=x / y(a-1 / a)$, and we obtain that

$$
\Omega_{s}^{\varphi} \cap \Sigma=\left\{\left(\begin{array}{cc}
a & (1+1 / y)(a+1 / a) \\
1 / a
\end{array}\right) \mid a>0\right\}
$$

(recall that $x^{2}+y^{2}=(\bar{w} w)^{2}=1$ ). Using the notation of (1.4), this means that $\Sigma_{S_{s} \varphi}=\Omega_{s}^{\varphi} \cap \Sigma=\Lambda(1+1 / y)$.

Applying (1.3) and (1.4), we infer from (3.5), (3.6) and (3.7):

(3.8) THEOREM. Assume that $\Sigma=\mathrm{SL}_{2} \mathbb{R}$ acts effectively and transitively on a 2-dimensional stable plane $\mathscr{S}$. If there is a $\Sigma$-equivariant embedding $(\pi, \lambda, 0)$ of $\mathscr{S}$ into $\mathscr{S}_{\mathbb{C}}$, then there is $w \in \mathbb{C}$ such that $w \bar{w}=1$ and $(w, w)$ belongs to the image of $\pi$. If $w^{2} \in \mathbb{R}$, then $\mathscr{S}$ is isomorphic with the punctured affine plane over $\mathbb{R} ;$ if $w^{2} \in \mathbb{C} \backslash \mathbb{R}$, then $\mathscr{S}$ is isomorphic with the punctured Strambach plane.

(3.9) FINAL REMARKS. (a) Since we fix the embedding (and thus the action) of $\Sigma$, we obtain the same subgeometry for choices $w \in\{u, v\}$ if, and only if, the point $(u, u)$ is contained in the orbit $(v, v) \Sigma$. Using the fact that $\Sigma$ acts transitively on $\mathbb{R}^{2} \backslash\{(0,0)\}$, one infers that this condition is equivalent to the condition that $u^{-1} v \in \mathbb{R}$. Under the additional assumption that $u \bar{u}=1=v \bar{v}$ this amounts to $u= \pm v$.

(b) One can show (see [1]) that for each continuous monomorphism $l: \Sigma=\mathrm{SL}_{2} \mathbb{R} \rightarrow \Delta=\mathrm{SL}_{2} \mathbb{C}$, the image $\Sigma^{l}$ is a conjugate of $\Sigma$ in $\Delta$. Thus in (3.2) the assumption that $\gamma=\mathbb{1}$ is not an essential restriction.

\section{REFERENCES}

1. Darmstadt, S. S. L., 'On complex Lie algebras with a simple real form', Seminar Sophus Lie, Darmstadt, Erlangen, Greifswald, Leipzig 1 (1991), 243-245. 
2. Löwen, R., 'Zweidimensionale stabile Ebenen mit nicht-auflösbarer Automorphismengruppe', Arch. Math. 41 (1983), 565-571.

3. Löwen, R., 'Actions of $\mathrm{Spin}_{3}$ on 4-dimensional stable planes', Geom. Dedicata 21 (1986), 1-12.

4. Strambach, K., 'Zur Klassifikation von Salzmann-Ebenen mit dreidimensionaler Kollineationsgruppe', Math. Ann. 179 (1968), 15-30.

5. Stroppel, M., 'A characterization of quaternion planes', Geom. Dedicata 36 (1990), 405-410.

6. Stroppel, M., 'Reconstruction of incidence geometries from groups of automorphisms', Arch. Math. 58 (1992), 621-624.

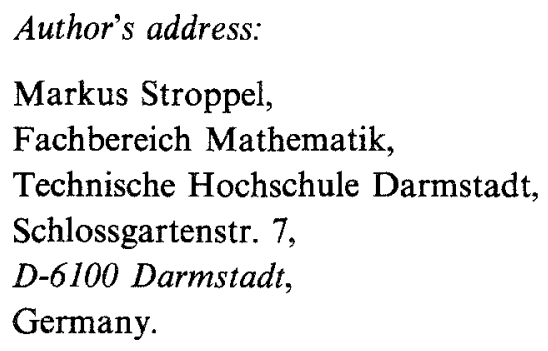

(Received, February. 12, 1992; revised version, May 5, 1992) 